\title{
Metal Halide Perovskites for Energy Applications
}

\author{
Wei Zhang ${ }^{\dagger}$, Giles E. Eperon, Henry J. Snaith* \\ Clarendon Laboratory, University of Oxford, Parks Road, Oxford OX1 3PU, UK
}

Correspondence and requests for materials should be addressed to H.J.S. (email: h.snaith1@physics.ox.ac.uk).

\footnotetext{
${ }^{\dagger}$ Present address: School of Chemistry, University of Lincoln, Beevor Street, Lincoln LN6 7DL, UK.
}

Abstract Exploring prospective materials for energy production and storage is one of the biggest challenges in this century. Solar energy is arguably the most important amongst various renewable energy resources, due to its wide availability and sustainability with lowest environmental impact. Metal halide perovskites have emerged as a class of semiconductor materials with unique properties including tuneable band gap, high absorption coefficient, broad absorption spectrum, high charge carrier mobility and long charge diffusion lengths, which enable a broad range of photovoltaic and optoelectronic applications. Since the first embodiment of perovskite solar cells showing a power conversion efficiency of $3.8 \%$, the device performance has now been boosted up to a certified $21 \%$ within a few years. In this perspective, we summarize differing forms of perovskite materials produced via various deposition procedures, focusing on their energy related applications, and discuss current challenges and possible solutions, with the aim of stimulating potential new applications.

\section{Introduction}

Metal halide perovskites, with the general formula of $A B X_{3}$ (where $A$ is a cation, $B$ a divalent metal ion, and $X$ a halide) are a class of semiconductors which have the potential to deliver cheaper and more efficient photovoltaics than silicon-based technology. Over the last 5 years, metal halide perovskites have attracted tremendous research effort, due to their unique optical and electronic properties. To date, they have been applied to fields including photovoltaics (PVs), light emitting diodes and solar to fuel energy conversion devices. In particular, the rapid advancement in PV efficiency has been accompanied by a deeper understanding of the fundamental properties of the materials and operational mechanisms of devices. Furthermore, recent progress with both nanocrystal and macroscopic single crystal growth and characterisation calls for a rationalisation of the different forms of perovskite semiconductors, beyond the widely-used polycrystalline thin films. 
In this perspective, we discuss exciting recent developments of perovskite materials in energy-related applications, and offer our opinion on the future course of the field. In addition, we will consider the prospects for perovskites in the broader energy picture: their potential roles in production, storage, and usage, and discuss the current challenges and potential solutions in these various areas.

\section{Versatile forms of metal halide perovskites}

A unique feature of metal halide perovskites, as compared to other traditional semiconductors such as crystalline $\mathrm{Si}$ and $\mathrm{GaAs}$, is their high crystallinity despite low temperature processing (sub $200{ }^{\circ} \mathrm{C}$ ). This enables processing on a range of substrates, and also versatile forms of perovskite materials - ranging from nanocrystals to macroscopic single crystals - to be created with ease. In this section, in order of physical scale, we describe various forms of perovskites, followed by a discussion of their fundamental properties and potential applications.

Nano-meso-micro structured perovskites A plethora of nano to meso to micro structured metal halide perovskites have recently been synthesised. Monodisperse colloidal nanocrystals (NCs) based on fully inorganic caesium lead halide perovskites $\left(\mathrm{CsPbX}_{3}, \mathrm{X}=\right.$ $\mathrm{Cl}, \mathrm{Br}$, I or mixture thereof) have been synthesised by reacting Cs-oleate with a $\mathrm{Pb}(\mathrm{II})$-halide in octadecene ${ }^{1}$. The resultant perovskite NCs show remarkably high (up to 90\%) photoluminescence quantum efficiency (PLQE), narrow emission line-widths (12-42 nm) and spectral tunability across the full visible spectrum $(410-700 \mathrm{~nm})$ through compositional modulations and the quantum confinement (Fig. 1a). As compared to metal chalcogenidebased quantum dots, perovskite NCs have a simpler chemical synthetic route and do not seem to require complex core-shell structures which have been a prerequisite for high PLQE from CdSe quantum dots. The achievement of $90 \%$ PLQE is similarly astonishing as the sudden rise in $\mathrm{PV}$ efficiency the photovoltaic field has witnessed.

Similarly, $\mathrm{CsPbBr}_{3}$ (Fig. 1b) and $\mathrm{CsPbl}_{3}$ nanowires (Fig. 1c) were prepared through a catalyst-free solution-phase synthesis ${ }^{2}$. Surprisingly, $\mathrm{CsPbX}_{3} \mathrm{NCs}$ crystallize into cubic phase at room temperature while the $\mathrm{CsPbX}_{3}$ nanowires show orthorhombic phase, highlighting the importance of synthetic conditions and size and shape of the nanostructures on the crystal phase transitions: in the bulk, these materials are not stable in the cubic perovskite structure at room temperature in ambient conditions, but convert into a nonperovskite orthorhombic phase ${ }^{3}$, less interesting for light emission and PV applications. The short-term stabilisation of the $\mathrm{CsPbl}_{3} \mathrm{NCs}$ in the cubic phase is very interesting, but requires 
further modification to deliver entirely structurally stable NCs. Notably, a similar strategy has been adopted for the synthesis of $\mathrm{MAPbX}_{3} \mathrm{NCs}^{4,5}$ and $\mathrm{MAPbX}_{3}$ nanorod arrays ${ }^{6}$ (Fig. 1d).

Other formats such as platelets ${ }^{7}$ (Fig. 1e) or microspheres ${ }^{8}$ (Fig. 1f) have also been fabricated, to exploit their novel structure-function properties for light emitting applications. We expect the activity in perovskite based colloidal crystals and crystal control on the nano, meso and micro scale to emerge into a field in its own right over the next few years; it is especially relevant to low-power light emitting technologies.

Polycrystalline thin films Polycrystalline perovskite thin films are the dominant format for PV applications. Unlike traditional semiconductors used for thin-film solar cells, perovskite thin films can be fabricated with similar properties via various deposition procedures including solution processing ${ }^{9-11}$, physical vapour phase deposition ${ }^{12}$ or combinations thereof ${ }^{13}$. The solution processing has advantages over vapour phase deposition in terms of relying on inexpensive deposition equipment, whilst the latter avoids the usage of often toxic solvents and allows the deposition of homogenous thin films with precisely tuneable thickness on arbitrary substrates.

The quality of the thin films, which is higly dependenent on the material purity and fabrication method, is of paramount importance for device performance and reproducibility. To date, tremendous efforts have been devoted to optimizing the perovskite crystallization and film formation in order to obtain highly crystalline and pinhole free perovskite thin films. Several review papers discuss the details of the differing growth methods ${ }^{14,15}$. Recently, perovskite thin films with full surface coverage and large polycrystalline domains (Fig 1g) have been achieved on large area by doctor-blade coating the precursor inks on hot substrates ${ }^{16}$, resulting in impressive device efficiencies.

A paradox which has emerged is the rationalisation between observations of non-radiative recombination at grain boundaries in polycrystalline thin films ${ }^{17}$, and remarkably high radiative efficiency in perovskite nanocrystals. The prior motivates evolving the perovskite thin films towards single crystal domains, whereas the latter indicates that perovskite crystal surfaces are not inherently defective, indicating the alternative possibility of developing polycrystalline thin films with negligible non-radiative recombination losses.

\section{Macroscopic single crystals}

Unlike the classical cooling-induced crystallization for growing $\mathrm{MAPbX}_{3}$ single crystals which is time-consuming ${ }^{18}$, a new strategy so-called "anti-solvent vapor-assisted crystallization" that slowly diffuses an antisolvent into the precursor solution was recently developed ${ }^{19}$. This enables the preparation of high-quality single crystals at room temperature. The high quality is reflected by low trap-state densities on the order of $10^{9}$ to $10^{10} \mathrm{~cm}^{-3}$, comparable to 
crystalline silicon grown at high temperature $\left(10^{8}-10^{15} \mathrm{~cm}^{-3}\right)$, and charge carrier diffusion lengths exceeding 10 micrometers have been estimated, in comparison to $\sim 1$ micrometer in a polycrystalline film ${ }^{20}$.

The methods described above rely on the decreased solubility of the perovskite precursor salts in the growth medium. There has recently been an unexpected discovery of an apparent "inverse solubility" behaviour for $\mathrm{MAPbX}_{3}$ precursors ${ }^{21,22}$. As the temperature of the solution is increased, large crystals are observed to precipitate from the solution. Upon subsequent cooling the crystals re-dissolve. Despite lack of understanding of the mechanism, however, this process has been capitalised upon to enable the rapid synthesis of various large perovskite crystals (Fig. 1h,i), providing a good platform for a range of optical and electrical characterizations ${ }^{22}$, and broad optoelectronic applications such as photodetectors ${ }^{23}$ and radiation energy harvesting ${ }^{24}$. It is our opinion however, that the greatest benefit of the single crystal work will be an added learning and understanding of crystallisation, which will be beneficial for reaching optimum properties of the polycrystalline thin films.

\section{Applications}

The versatility of the material compositions and processing routes, in combination with the favourable optical and electrical properties, opens a new avenue for emerging applications of metal halide perovskites. In this section, several applications of metal halide perovskites including PVs, light-emission and solar energy storage will be discussed with a motivation to simulate potential new applications.

Photovoltaics (PVs) As discussed previously ${ }^{14,15}$, perovskites were originally viewed as a curious replacement to dye molecules in mesoscopic sensitized solar cells, due to its high absorption coefficient and broad absorption spectrum (Fig. 2a) ${ }^{9}$. However, it was quickly realized that perovskites are unique semiconductor materials differing from dye molecules and other organic absorbers, and more akin to the inorganic semiconductors for PV applications, such as $\mathrm{Si}$ or GaAs. Like these materials, perovskites have long carrier diffusion length ${ }^{20}$ and remarkable performance in planar heterojunction architectures (Fig. $2 b)^{12}$, where an intrinsic $(I)$ perovskite solid layer is sandwiched between an $n$-type electron collection layer (typically compact $\mathrm{TiO}_{2}$ ) and $p$-type hole collection layer (typically SpiroOMeTAD $)^{12}$. This structure has led to current density-voltage $(J-V)$ curve measured power conversion efficiencies (PCE) of up to $19.4 \%$ so far $^{25}$.

One "inconvenience" with this " $n-i-p$ " structure is that the $J-V$ curves exhibit a large hysteresis, which has been widely discussed ${ }^{26,27}$. It is now apparent that hysteresis 
originates from a coincidence of electronic defects and mobile ionic species ${ }^{28}$, and is not due to ferroelectricity ${ }^{29}$. To reduce this hysteresis, a very thin layer of mesoporous $\mathrm{TiO}_{2}$ (around $150 \mathrm{~nm}$ ) has been preserved in the highest efficiency devices reported thus far (Fig. 2c) ${ }^{30}$. We believe the active role of this thin mesoporous layer is to offer a higher surface area at the contact for making more favourable charge extraction, and to enable electron accumulation in the perovskite within this region, which acts to fill the electronic trap sites at this interface and inhibit accumulation of negative ionic charge.

An alternative solution for reducing hysteresis is to exchange the $n$-type compact $\mathrm{TiO}_{2}$ layer with an organic $n$-type collection layer and develop so-called inverted $p$-i-n perovskite cell by combing an organic $p$-type collection layer (Fig. $2 \mathrm{~d})^{31}$; it has been shown that cells with fullerene organic $n$-type charge collection layers exhibit negligible hysteresis in their $\mathrm{J}-\mathrm{V}$ curves $^{32}$. We interpret this to indicate that a large surface defect density is generated at the interface between the perovskite and compact $\mathrm{TiO}_{2}$, likely during perovskite crystallisation, whereas the organic materials appear not to induce such a high density of defects ${ }^{32}$. In addition to being hysteresis-free, such cells inherit the advantages of organic PVs: lowtemperature, solution processing and ease of tuning the energy level alignment at the interfaces. In this architecture a PCE of $19.4 \%$ has recently been reported ${ }^{25}$, showing the promise of this device structure. Based on its advantages, we predict that the organiccontacted structure will surpass the efficiency of the $\mathrm{TiO}_{2}$ based cells over the next year.

Multi-junction hybrid solar cells based on silicon or copper indium gallium selenide (CIGS) and perovskites are a very promising route to deliver a higher efficiency, cost-effective solar technology than that will compete favourably with today's technologies, and we believe this application is likely to be the first commercial appearance of the perovskite solar cells ${ }^{33,34}$. Here however, we will briefly discuss the applications of perovskite solar cells which can go beyond that feasible with crystalline silicon.

Key non-ideal properties of wafer based silicon PV are the requirement for a relatively heavy rigid format, and lack of aesthetic versatility, especially considering semi-transparent applications. In contrast, perovskite PVs can be processed on a variety of substrates via either solution or vapour phase processing, and have already delivered very high efficiency on a flexible format. Ultra-light weight perovskite solar cells with a power to weight ratio of 23 $\mathrm{W} / \mathrm{g}$, compared to that of space-rated silicon at $\sim 1 \mathrm{~W} / \mathrm{g}$, have been recently used to power a model aeroplane (Fig. 3a) ${ }^{35}$. This is a fun illustration of the future technologies which may be enabled by extremely light-weight high-power perovskite PVs; in particular this will be of interest for space applications, the air transportation industry and military.

In addition to flexibility, perovskite PVs can also be fabricated to be semi-transparent (Fig. $3 b)^{36}$, colourful (Fig. 3c $)^{37}$ or even integrated into photovoltachromic devices ${ }^{38}$ for building integrated photovoltaics (BIPVs) applications. 
Light-emitting devices for reduced energy consumption Efficient solid-state lighting based on inorganic (primarily GaN) light-emitting diodes (LEDs) has revolutionised the lighting industry and is set to displace all incandescent and fluorescent lighting over the next decade. A key driver for the lighting industry is efficient and colour tuneable emitters. An ideal light absorber for PVs, like perovskite, should also be an ideal light emitter, where all recombination is ultimately radiative according to the Shockley-Quieisser treatment ${ }^{39}$. Unlike the early embodiments of LEDs based on layer-structured perovskites, showing electroluminescence only at extremely low temperatures, LEDs based on metal halide perovskite thin films such as $\mathrm{MAPbl}_{3-x} \mathrm{Cl}_{x}$ (meaning perovskite prepared from $\mathrm{Cl}$-containing precursors) or $\mathrm{MAPbl}_{3-\mathrm{x}} \mathrm{Br}_{\mathrm{x}}$, as depicted by Fig. 3d, exhibit strong PL emission and tuneable emission spectra at room temperature by varying the composition of the perovskite ${ }^{40}$. Very recently, an extremely impressive external quantum efficiency of $8.53 \%$ based on $\mathrm{MAPbBr}_{3}$ has been reported, achieved by optimising film morphology and material composition ${ }^{41}$. Further gains may be made by employing the previously discussed perovskite nanocrystals (Fig. 2a), leveraging their higher PLQE than the thin-film counterparts.

The narrow emission spectrum and high PLQE of metal halide perovskites also open up possibilities for fabrication of low-cost lasers. Previous prototypes using polycrystalline perovskite thin films suffer from a relatively high threshold and low quality factor, possibly due to the limited crystallinity and high defect density ${ }^{42}$. A striking breakthrough was recently made by using solution-processed perovskite single-crystal nanowires showing a near $100 \%$ PLQE with a low threshold, high quality factor and wide spectral tunability (Fig. 3e) ${ }^{43}$. This highlights the importance of controlling the crystallinity and structural defects of the perovskite materials in achieving high performance light emitting devices.

Bearing these considerations in mind, it seems likely that a divergence between the PV and light emitting research will be one of scale of the perovskite crystal domains, where isolated nanocrystals in a host may prove to be more advantageous for LEDs or lasers, in contrast to large crystalline domains in homogeneous thin films for the PV research. In our opinion, understanding the origin of non-radiative recombination in PL emission and finding routes to reduce such recombination pathways is the major challenge for further boosting the performance of light emitting devices. Additionally, developing structurally and thermally stable crystals of the appropriate band-gaps also needs realisation.

Solar fuels for energy storage Deployment of solar energy usually requires energy storage. One option is to convert solar energy into chemical energy through artificial photosynthesis, producing so-called "solar fuels". Typically, solar fuels are obtained by reducing protons into 
hydrogen through water splitting or by reducing carbon dioxide into organic chemicals. A perovskite-based solar-to-hydrogen conversion system has been built by connecting two perovskite solar cells in series, providing a high enough voltage to drive water-splitting. This achieved a solar to hydrogen conversion efficiency of $12.0 \%$ (Fig. $3 f)^{44}$, close to that obtained by GalnP/GaAs tandem cells (12.4\%) but much cheaper ${ }^{45}$. Sunlight-driven $\mathrm{CO}_{2}$ reduction was recently achieved ${ }^{46}$ (Fig. $3 \mathrm{~g}$ ), achieving a solar-to-CO efficiency of $6.5 \%$. The key to the good performance of these conversion systems is the low loss-in-voltage achieved by the perovskite solar cells. It becomes realistic to achieve a solar-to-fuel efficiency over $20 \%$ in next few years for the production of $\mathrm{H}_{2}$ or organic chemicals by combining more efficient perovskite tandem solar cells with more effective catalysts.

\section{Challenges and Opportunity}

\section{Understanding what makes $\mathrm{MAPbX}_{3}$ perovskites such good semiconductors. In} addition to the well-known properties of perovskites, a unique feature that makes perovskites so good for PV applications relies on the fact that the energy of many electronic defects is expected to be close to the continuum of states within the bands, hence not detrimental for performance ${ }^{47}$.

Despite this, there is still ample room to improve device performance by further reducing the densities of defects which are responsible for trap-assisted recombination. The origin of these trap related defects is currently unknown, but the densities of defects have substantial effect on the charge carrier recombination kinetics and device performance in the regime of solar sunlight intensity ${ }^{48}$. Furthermore, the defects appear to largely reside in the regions near the grain boundaries which serve as non-radiative recombination centres ${ }^{17}$. Encouragingly, through chemical passivation post-treatment, those defective regions can be somewhat "lit-up" again. Besides better control of crystallisation and passivation of defects, making larger crystal grains should be another way to reduce the grain boundaries and ensuing defect densities. However, there have not yet been numerous correlations between increasing grain size and improved material and device properties ${ }^{49}$. In addition, it is not clear if "grains" as observed in SEM images are single crystal grains or polycrystalline beneath the surface ${ }^{16,49}$. Combined with the observation of high PLQE from nanoscale crystals, this leads to an important and currently unanswered question: do we want large crystals, or well controlled, and potentially well-passivated small crystals in the ideal perovskite thin film? It is our opinion that it will not be necessary to move towards singlecrystal thin films, but optimal thin films may consists of crystalline domains with dimensions slightly larger than but on the order of the film thickness, with well controlled and passivated grain boundaries and surfaces. 
Perovskites beyond $\mathrm{MAPbX}_{3}$ ? One primary challenge of $\mathrm{MAPbX}_{3}$ in $\mathrm{PV}$ and other applications is the inherent instability of materials toward moisture, air and heat ${ }^{50}$. A means to improve the stability is replacing $\mathrm{MA}$ with $\mathrm{FA}^{51}$ or $\mathrm{Cs}^{3}$ or mixture thereof ${ }^{33}$. Another possible route is based on the homologous two-dimensional materials, where sheets of perovskite octahedra are separated by layers of larger organics ${ }^{52}$. Although they have less ideal properties for solar cell power generation, the long chain organic cations afford a degree of hydrophobicity, and superior moisture stability has been reported ${ }^{53,54}$. These materials should also be very promising for luminescent applications due to their strong emission characteristics ${ }^{55}$.

Toxicity is the other obvious challenge for lead-based perovskites, due to the possibility of lead leaching into the environment ${ }^{56}$. Clearly, a lead-free perovskite material would allay public concern. The early embodiments of this are the Sn-based perovskites ${ }^{57},{ }^{58}$, showing narrower band gaps and higher mobilities than lead-based perovskites, with typical PCEs in solar cells of around 6.0\%. Perovskite materials based on Ge were also reported recently ${ }^{59,60}$, showing PCEs around $0.2 \%{ }^{60}$. Although Sn- or Ge-based perovskites should be less toxic than the lead counterparts, their poor chemical stability due the oxidation of $\mathrm{Sn}$ or $\mathrm{Ge}$ currently restricts their PV applications. If their stability can be enhanced, these materials could become useful for real applications. An alternative choice could be mixed $(\mathrm{Sn}, \mathrm{Pb})$ or $(\mathrm{Ge}, \mathrm{Pb})$ perovskites, for example, MASn $\mathrm{Pb}_{1-x} \mathrm{I}_{3}$ or $\mathrm{MAGe}_{x} \mathrm{~Pb}_{1-\mathrm{x}} \mathrm{I}_{3}$, where the amount of $\mathrm{Pb}$ is reduced and the stability is maintained, meanwhile offering new possibility of tuning the optical and optoelectrical properties.

Opportunities as energy storage materials Perovskite solar cells devices exhibit currentvoltage hysteresis ascribed to a combination of ionic motion and electronic traps within the perovskite. This has been shown to stem from a low activation energy - and thus high ionic conductivity - for vacancies or interstitials in the material ${ }^{61}$. This then leads us to ask, can we make use of this solid-state high ionic conductivity in any form of device? The obvious candidate is in energy storage: if we can move charged ions or defects around in the material, this may be a good way to store charge. Here we briefly consider the two most promising embodiments: batteries and supercapacitors.

Perovskites in batteries The most popular batteries in use today are ion batteries, predominantly lithium-ion. These comprise an ion-conducting electrolyte connecting two 
lithium-ion-storing electrodes. Lithium ions are driven into the anode by applying bias, intercalating in the material, with the positive charge and compensated by electrons ${ }^{62}$. Under external load, the battery discharges: the lithium ions return to the cathode via the electrolyte and a corresponding flow of electrons is driven around the external circuit, powering the load.

An electrode must be capable of reversibly storing as many ions as possible. In a lithium-ion battery $^{63-65}$, the cathode is generally the limiting electrode, with state-of-the-art storage capacities for archetypal cathodes $\mathrm{LiCoO}_{2}, \mathrm{LiFePO}_{4}$, and $\mathrm{LiMn}_{2} \mathrm{O}_{4}$ being around 150-170 $\mathrm{mAhg}^{-1}$. Common anodes include graphite ${ }^{64}$, with a storage capacity of $370 \mathrm{mAhg}^{-1}$. The current challenges are improving electrode stability, capacity and lowering cost. Perovskites, may be able to offer both improved capacity and lower cost.

Much work has been carried out on similar materials for battery anodes and cathodes; particularly similar materials are metal-organic frameworks ${ }^{66}$, which have been used as lithium-ion cathodes $\left(75 \mathrm{mAhg}^{-1}\right)$ and anodes $\left(510 \mathrm{mAhg}^{-1}\right)$, and the pigment Prussian blue ${ }^{67}$, which has been posited as a cathode for sodium-ion batteries (up to $100 \mathrm{mAhg}^{-1}$ ). We show the structures of some electrode materials in Fig. 4. We note that many materials have similar corner-sharing structures to the $\mathrm{ABX}_{3}$ perovskites, indicating that this structure may be suitable for ion storage. Supporting this postulation, there has recently been a first report of $\mathrm{MAPbX}_{3}$ perovskites used as lithium-storing anode materials with storage capacity of $330 \mathrm{mAhg}^{-1}$, which is competitive with the state-of-the-art anodes ${ }^{68}$. It remains to be seen whether the perovskites can play a role here in the performance-limiting cathode too, but the lithium storage capability reported is certainly promising.

We point out that there remains an open question of where the lithium is actually stored via intercalation or at the surface. Furthermore, the anode deteriorated quickly, indicating that stability may be a challenge. In our opinion, more promise may lie in the layered 2D perovskites, which have clearer intercalation paths due to more space in the structure.

Perovskites as supercapacitors Supercapacitors have a much higher capacity than conventional parallel-plate capacitors (up to $\sim 30 \mathrm{Wh} / \mathrm{kg}$ ) and a faster discharge rate than batteries $^{62}$. They typically consist of two electrodes contacted by an electrolyte, rather than a simple dielectric as in a parallel-plate capacitor. The key difference is that ions in the electrolyte can move in response to a field. Energy is stored in an electric double layer at the surface of the electrode; because it is thin the capacitance is high. The other difference is that the electrode surface area is orders of magnitude larger than a flat surface; materials such as porous carbon are often used. 
We postulate that perovskites could effectively replace the electrodes or electrolyte in a supercapacitor, taking advantage of the high surface area and good ionic mobility to provide high charge storage. lonic motion within the material itself has been determined to provide capacitance of $\sim 1.8 \mathrm{mFcm}^{-2}$ in a $350 \mathrm{~nm}$ thick $\mathrm{MAPbl}_{3}$ film ${ }^{29}$ in a parallel-plate capacitor (electrostatically). If we consider an operating voltage of $1 \mathrm{~V}$, a capacitor of these dimensions would store $\sim 2 \times 10^{-6} \mathrm{Wh} / \mathrm{kg}$, capacitance of $12.6 \mathrm{~F} / \mathrm{g}$. We can then make a rough calculation of the energy storage for a supercapacitor consisting of perovskite contacting high surface area carbon (surface area of $\left.1000 \mathrm{~m}^{2} / \mathrm{g}\right)^{69}$, assuming that the previously measured capacitance ${ }^{29}$ is proportional to the surface area, not the volume, and a 1:1 ratio of perovskite to carbon by weight. The capacitance of such an interface will be $9000 \mathrm{~F} / \mathrm{g}$ or $1250 \mathrm{Wh} / \mathrm{kg}$, which looks very promising as compared to a typical carbon electrode in contact with liquid electrolyte (around $100 \mathrm{~F} / \mathrm{g}$ ) ${ }^{69}$.

We note that this rough calculation assumes that all of the very large interface area experiences the same capacitance as the parallel-plate capacitor; this would undoubtedly be complicated by interface energetics and practical considerations. However, it seems likely to us that the perovskites could create a new type of supercapacitor, where the ionic motion allows them to act similarly to an electrolyte and the high capacitance similar to an electrode - we envisage that a perovskite film sandwiched between two high surface area electrodes to be a possible iteration (Fig. 4).

\section{Concluding remarks}

This perspective has endeavoured to highlight the remarkably versatility of the metal halide perovskite semiconductor family. Increasingly exciting applications are being realised and the perovskites appear poised to excel in many of them. For the dominant application of perovskites PVs, we expect that the PCE will continue to progress toward the ShockleyQuisser limit, by the combination of perovskite crystal orientation, surface and quality control, to compete with $\mathrm{Si}$ in the near future. Perovskite PVs will also see further applications in the solar fuel area, leveraging their high performance and low cost to reach new conversion records. We postulate that the rapidly advancing field of perovskites in light emission may begin to diverge from PVs in terms of the morphology of the materials used, as this field pushes closer towards highly efficient energy-saving LEDs and lasers. Herein, we propose perovskite-based energy storage as a potential new and exciting area of research, envisaging perovskite batteries and capacitors as efficient and cheap storage devices. We can also envisage the promise of the perovskites reaching into other areas not discussed in 
this perspective in the next few years, making perovskites a fantastic family of materials surpassing the dominant semiconductors in the market ( $\mathrm{Si}, \mathrm{CdTe}$ or $\mathrm{GaAs}$ ) for a broad range of energy related applications.

\section{References}

1 Protesescu, L. et al. Nanocrystals of Cesium Lead Halide Perovskites ( $\mathrm{CsPbX} 3, \mathrm{X}=\mathrm{Cl}, \mathrm{Br}$, and I): Novel Optoelectronic Materials Showing Bright Emission with Wide Color Gamut. Nano letters 15, 3692-3696 (2015).

2 Zhang, D., Eaton, S. W., Yu, Y., Dou, L. \& Yang, P. Solution-Phase Synthesis of Cesium Lead Halide Perovskite Nanowires. Journal of the American Chemical Society 137, 9230-9233 (2015).

3 Eperon, G. E. et al. Inorganic caesium lead iodide perovskite solar cells. Journal of Materials Chemistry A 3, 19688-19695 (2015).

4 Schmidt, L. C. et al. Nontemplate synthesis of $\mathrm{CH} 3 \mathrm{NH} 3 \mathrm{PbBr} 3$ perovskite nanoparticles. Journal of the American Chemical Society 136, 850-853 (2014).

5 Jang, D. M. et al. Reversible Halide Exchange Reaction of Organometal Trihalide Perovskite Colloidal Nanocrystals for Full-Range Band Gap Tuning. Nano letters 15, 5191-5199 (2015).

6 Wong, A. B. et al. Growth and Anion Exchange Conversion of CH3NH3PbX3 Nanorod Arrays for Light-Emitting Diodes. Nano letters 15, 5519-5524 (2015).

7 Zhang, Q., Ha, S. T., Liu, X., Sum, T. C. \& Xiong, Q. Room-temperature near-infrared high-Q perovskite whispering-gallery planar nanolasers. 14, 5995-6001 (2014).

8 Sutherland, B. R., Hoogland, S., Adachi, M. M., Wong, C. T. \& Sargent, E. H. Conformal organohalide perovskites enable lasing on spherical resonators. ACS nano 8, 10947-10952 (2014).

$9 \quad \mathrm{Kim}, \mathrm{H}$.-S. et al. Lead iodide perovskite sensitized all-solid-state submicron thin film mesoscopic solar cell with efficiency exceeding 9\%. Scientific reports 2, 591 (2012).

10 Lee, M. M., Teuscher, J., Miyasaka, T., Murakami, T. N. \& Snaith, H. J. Efficient hybrid solar cells based on meso-superstructured organometal halide perovskites. Science 338, 643-647 (2012).

11 Burschka, J. et al. Sequential deposition as a route to high-performance perovskitesensitized solar cells. Nature 499, 316-319 (2013).

12 Liu, M., Johnston, M. B. \& Snaith, H. J. Efficient planar heterojunction perovskite solar cells by vapour deposition. Nature 501, 395-398 (2013).

13 Chen, Q. et al. Planar heterojunction perovskite solar cells via vapor-assisted solution process. Journal of the American Chemical Society 136, 622-625 (2013).

14 Stranks, S. D., Nayak, P. K., Zhang, W., Stergiopoulos, T. \& Snaith, H. J. Formation of Thin Films of Organic - Inorganic Perovskites for High - Efficiency Solar Cells. Angewandte Chemie International Edition 54, 3240-3248 (2015).

15 Song, T.-B. et al. Perovskite solar cells: film formation and properties. Journal of Materials Chemistry A 3, 9032-9050 (2015).

16 Deng, Y. et al. Scalable fabrication of efficient organolead trihalide perovskite solar cells with doctor-bladed active layers. Energy \& Environmental Science 8, 1544-1550 (2015).

17 de Quilettes, D. W. et al. Impact of microstructure on local carrier lifetime in perovskite solar cells. Science 348, 683-686 (2015).

18 Poglitsch, A. \& Weber, D. Dynamic disorder in methylammoniumtrihalogenoplumbates (II) observed by millimeter - wave spectroscopy. The Journal of chemical physics $87,6373-6378$ (1987). 
19 Shi, D. et al. Low trap-state density and long carrier diffusion in organolead trihalide perovskite single crystals. Science 347, 519-522 (2015).

20 Stranks, S. D. et al. Electron-hole diffusion lengths exceeding 1 micrometer in an organometal trihalide perovskite absorber. Science 342, 341-344 (2013).

21 Kadro, J. M., Nonomura, K., Gachet, D., Grätzel, M. \& Hagfeldt, A. Facile route to freestanding CH3NH3PbI3 crystals using inverse solubility. Scientific Reports 5, 11654 (2015). Saidaminov, M. I. et al. High-quality bulk hybrid perovskite single crystals within minutes by inverse temperature crystallization. Nature communications 6, 7586 (2015).

23 Fang, Y., Dong, Q., Shao, Y., Yuan, Y. \& Huang, J. Highly narrowband perovskite single-crystal photodetectors enabled by surface-charge recombination. Nature Photonics, doi:10.1038/nphoton.2015.1156 (2015).

24 Dong, Q. et al. Electron-hole diffusion lengths $>175 \mu \mathrm{m}$ in solution-grown $\mathrm{CH} 3 \mathrm{NH} 3 \mathrm{Pbl} 3$ single crystals. Science 347, 967-970 (2015).

25 Shao, Y., Yuan, Y. \& Huang, J. Correlation of energy disorder and open-circuit voltage in hybrid perovskite solar cells. Nature Energy 1, 15001 (2016).

26 Snaith, H. J. et al. Anomalous hysteresis in perovskite solar cells. The Journal of Physical Chemistry Letters 5, 1511-1515 (2014).

27 Unger, E. et al. Hysteresis and transient behavior in current-voltage measurements of hybrid-perovskite absorber solar cells. Energy \& Environmental Science 7, 3690-3698 (2014). van Reenen, S., Kemerink, M. \& Snaith, H. J. Modeling Anomalous Hysteresis in Perovskite Solar Cells. The Journal of Physical Chemistry Letters 6, 3808-3814 (2015).

29 Beilsten-Edmands, J., Eperon, G., Johnson, R., Snaith, H. \& Radaelli, P. Non-ferroelectric nature of the conductance hysteresis in $\mathrm{CH} 3 \mathrm{NH} 3 \mathrm{Pbl} 3$ perovskite-based photovoltaic devices. Applied Physics Letters 106, 173502 (2015).

30 Yang, W. S. et al. High-performance photovoltaic perovskite layers fabricated through intramolecular exchange. Science 348, 1234-1237 (2015).

31 Heo, J. H., Han, H. J., Kim, D., Ahn, T. K. \& Im, S. H. Hysteresis-less inverted CH 3 NH 3 Pbl 3 planar perovskite hybrid solar cells with $18.1 \%$ power conversion efficiency. Energy \& Environmental Science 8, 1602-1608 (2015).

32 Shao, Y., Xiao, Z., Bi, C., Yuan, Y. \& Huang, J. Origin and elimination of photocurrent hysteresis by fullerene passivation in $\mathrm{CH} 3 \mathrm{NH} 3 \mathrm{PbI} 3$ planar heterojunction solar cells. Nature communications 5, 5784 (2014).

33 McMeekin, D. P. et al. A mixed-cation lead mixed-halide perovskite absorber for tandem solar cells. Science 351, 151-155 (2016).

34 Fu, F. et al. Low-temperature-processed efficient semi-transparent planar perovskite solar cells for bifacial and tandem applications. Nature communications 6 (2015).

35 Kaltenbrunner, M. et al. Flexible high power-per-weight perovskite solar cells with chromium oxide-metal contacts for improved stability in air. Nature materials 14, 1032-1039 (2015).

36 Eperon, G. E., Burlakov, V. M., Goriely, A. \& Snaith, H. J. Neutral color semitransparent microstructured perovskite solar cells. ACS nano 8, 591-598 (2013).

37 Zhang, W. et al. Highly Efficient Perovskite Solar Cells with Tunable Structural Color. Nano letters 15, 1698-1702 (2015).

38 Cannavale, A. et al. Perovskite photovoltachromic cells for building integration. Energy \& Environmental Science 8, 1578-1584 (2015).

39 Miller, O. D., Yablonovitch, E. \& Kurtz, S. R. Strong internal and external luminescence as solar cells approach the Shockley-Queisser limit. Photovoltaics, IEEE Journal of 2, 303-311 (2012).

40 Tan, Z.-K. et al. Bright light-emitting diodes based on organometal halide perovskite. Nature nanotechnology 9, 687-692, doi:10.1038/nnano.2014.149 (2014).

41 Cho, H. et al. Overcoming the electroluminescence efficiency limitations of perovskite lightemitting diodes. Science 350, 1222-1225 (2015). 
42 Deschler, F. et al. High photoluminescence efficiency and optically pumped lasing in solution-processed mixed halide perovskite semiconductors. The Journal of Physical Chemistry Letters 5, 1421-1426 (2014).

43 Zhu, H. et al. Lead halide perovskite nanowire lasers with low lasing thresholds and high quality factors. Nature materials 14, 636-642 (2015).

44 Luo, J. et al. Water photolysis at $12.3 \%$ efficiency via perovskite photovoltaics and Earthabundant catalysts. Science 345, 1593-1596 (2014).

45 Khaselev, O. \& Turner, J. A. A monolithic photovoltaic-photoelectrochemical device for hydrogen production via water splitting. Science 280, 425-427 (1998).

46 Schreier, M. et al. Efficient photosynthesis of carbon monoxide from $\mathrm{CO} 2$ using perovskite photovoltaics. Nature communications 6, 7236 (2015).

47 Yin, W.-J., Shi, T. \& Yan, Y. Superior Photovoltaic Properties of Lead Halide Perovskites: Insights from First-Principles Theory. The Journal of Physical Chemistry C 119, 5253-5264 (2015).

48 Stranks, S. D. et al. Recombination Kinetics in Organic-Inorganic Perovskites: Excitons, Free Charge, and Subgap States. Physical Review Applied 2, 034007 (2014).

49 Nie, W. et al. High-efficiency solution-processed perovskite solar cells with millimeter-scale grains. Science 347, 522-525 (2015).

50 Leijtens, T. et al. Stability of Metal Halide Perovskite Solar Cells. Advanced Energy Materials 5,1500963 (2015).

51 Eperon, G. E. et al. Formamidinium lead trihalide: a broadly tunable perovskite for efficient planar heterojunction solar cells. Energy \& Environmental Science 7, 982-988 (2014).

52 Mitzi, D. B. Synthesis, structure, and properties of organic - inorganic perovskites and related materials. Progress in Inorganic Chemistry, Volume 48, 1-121 (2007).

53 Smith, I. C., Hoke, E. T., Solis - Ibarra, D., McGehee, M. D. \& Karunadasa, H. I. A Layered Hybrid Perovskite Solar - Cell Absorber with Enhanced Moisture Stability. Angewandte Chemie 126, 11414-11417 (2014).

54 Cao, D. H., Stoumpos, C. C., Farha, O. K., Hupp, J. T. \& Kanatzidis, M. G. 2D Homologous Perovskites as Light-Absorbing Materials for Solar Cell Applications. Journal of the American Chemical Society 137, 7843-7850 (2015).

55 Dou, L. et al. Atomically thin two-dimensional organic-inorganic hybrid perovskites. Science 349, 1518-1521 (2015).

56 Babayigit, A., Ethirajan, A., Muller, M. \& Conings, B. Toxicity of organometal halide perovskite solar cells. Nature Materials 15, 247-251 (2016).

57 Noel, N. K. et al. Lead-free organic-inorganic tin halide perovskites for photovoltaic applications. Energy \& Environmental Science 7, 3061-3068 (2014).

58 Hao, F., Stoumpos, C. C., Cao, D. H., Chang, R. P. \& Kanatzidis, M. G. Lead-free solid-state organic-inorganic halide perovskite solar cells. Nature Photonics 8, 489-494 (2014).

59 Stoumpos, C. C. et al. Hybrid Germanium lodide Perovskite Semiconductors: Active Lone Pairs, Structural Distortions, Direct and Indirect Energy Gaps and Strong Nonlinear Optical Properties. Journal of the American Chemical Society 137, 6804-6819 (2015). Thirumal, K. et al. Lead-free Germanium lodide Perovskite Materials for Photovoltaic Application. Journal of Materials Chemistry A, DOI: 10.1039/C1035TA05741H (2015).

61 Eames, C. et al. Ionic transport in hybrid lead iodide perovskite solar cells. Nature communications 6, 7497 (2015).

62 Winter, M. \& Brodd, R. J. What are batteries, fuel cells, and supercapacitors? Chemical reviews 104, 4245-4270 (2004).

63 Erickson, E. M., Ghanty, C. \& Aurbach, D. New Horizons for Conventional Lithium Ion Battery Technology. The Journal of Physical Chemistry Letters 5, 3313-3324 (2014).

64 Liu, C., Li, F., Ma, L. P. \& Cheng, H. M. Advanced materials for energy storage. Advanced Materials 22, E28-E62 (2010). 
65 Islam, M. S. \& Fisher, C. A. Lithium and sodium battery cathode materials: computational insights into voltage, diffusion and nanostructural properties. Chemical Society Reviews 43, 185-204 (2014).

66 Combelles, C., Yahia, M. B., Pedesseau, L. \& Doublet, M.-L. Fe II/Fe III mixed-valence state induced by Li-insertion into the metal-organic-framework Mil53 (Fe): A DFT+ U study. Journal of Power Sources 196, 3426-3432 (2011).

67 Lu, Y., Wang, L., Cheng, J. \& Goodenough, J. B. Prussian blue: a new framework of electrode materials for sodium batteries. Chemical communications 48, 6544-6546 (2012).

68 Xia, H.-R., Sun, W.-T. \& Peng, L.-M. Hydrothermal synthesis of organometal halide perovskites for Li-ion batteries. Chemical Communications 51, 13787-13790 (2015).

69 Zhang, L. L. \& Zhao, X. Carbon-based materials as supercapacitor electrodes. Chemical Society Reviews 38, 2520-2531 (2009).

70 Malinkiewicz, O. et al. Perovskite solar cells employing organic charge-transport layers. Nature Photonics 8, 128-132 (2014).
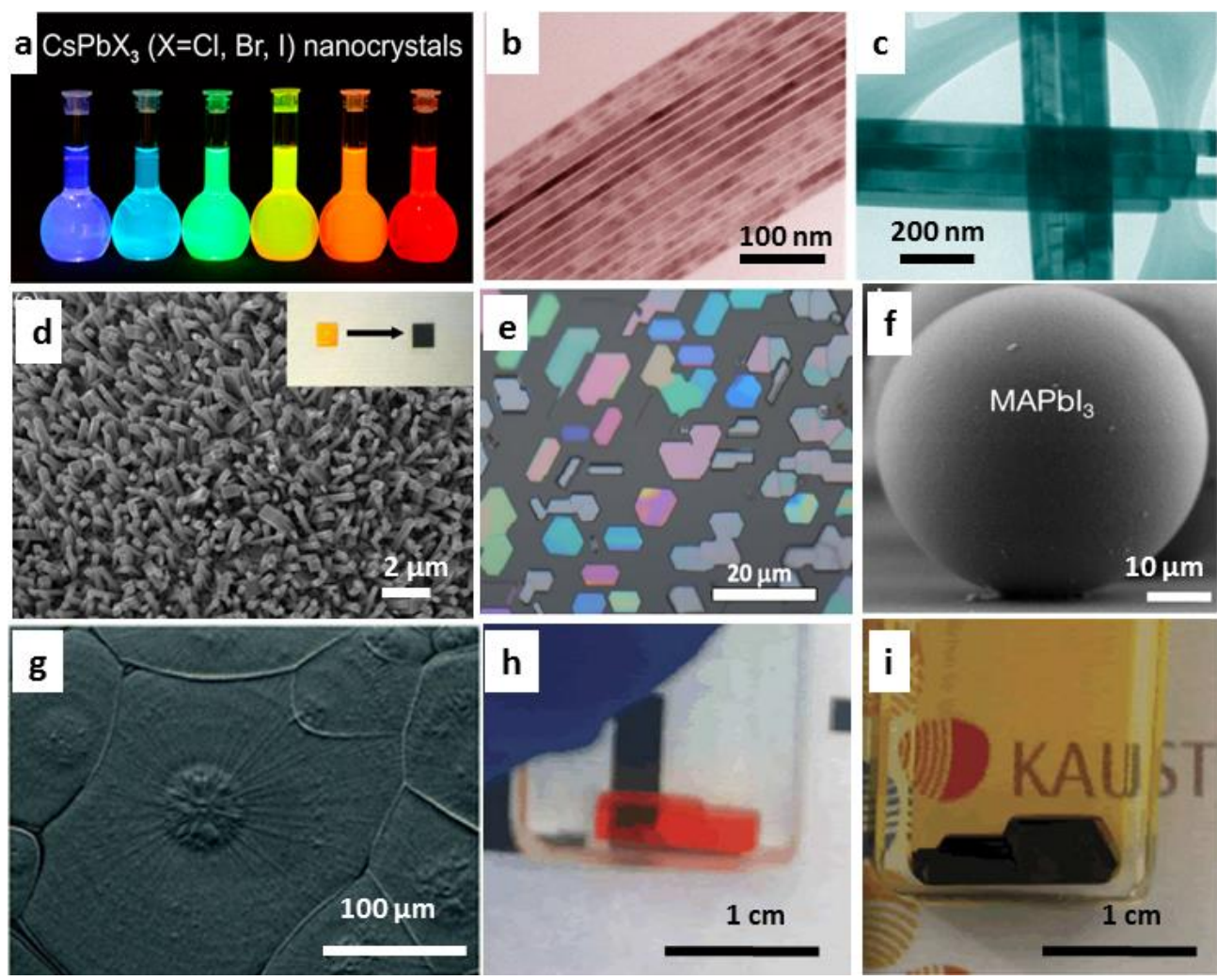

Figure 1. Versatile forms of metal halide perovskites. a, colloidal solutions of $\mathrm{CsPbX}_{3}(\mathrm{X}=$ $\mathrm{Cl}, \mathrm{Br}, \mathrm{I})$ nanocrystals in toluene under UV $\operatorname{lamp}^{1}(\lambda=365 \mathrm{~nm}) . \mathbf{b}, \mathbf{c}$, scanning electron microscopy (SEM) images of $\mathrm{CsPbBr}_{3}$ (b) and $\mathrm{CsPbl}_{3}$ (c) nanowires grown by solutionphase synthesis ${ }^{2}$. d, $\mathrm{CH}_{3} \mathrm{NH}_{3} \mathrm{Pbl}_{3}$ nanorod array formed by vapor phase anion exchange of $\mathrm{CH}_{3} \mathrm{NH}_{3} \mathrm{PbBr}_{3}$ nanorod array. The inset shows a photograph of a converted chip ${ }^{6}$. e, an optical image of $\mathrm{CH}_{3} \mathrm{NH}_{3} \mathrm{Pbl}_{3}$ platelets prepared by vapor phase synthesis ${ }^{7}$. $\mathbf{f}, \mathrm{SEM}$ image of a $\mathrm{SiO}_{2}$ microsphere conformally coated with $\mathrm{CH}_{3} \mathrm{NH}_{3} \mathrm{Pbl}_{3}$ via atomic layer deposition ${ }^{8}$. g, a SEM image of $\mathrm{CH}_{3} \mathrm{NH}_{3} \mathrm{Pbl}_{3}$ thin film with large polycrystalline domains prepared by doctor- 
blade coating on large area ${ }^{16} . \mathbf{h}$, i, shape-controlled single crystals of $\mathrm{MAPbBr}_{3}(\mathrm{~h})$ and $\mathrm{MAPbl}_{3}$ (i) formed by inverse temperature crystallization ${ }^{22}$. Figures reproduced with permission from: a, ref 1, ACS; b,c, ref 2, ACS; d, ref 6, ACS; e, ref 7, Wiley; f, ref 8, ACS; g, ref 16, RSC; $\mathbf{h}$, i, ref 22, Nature Publishing Group.

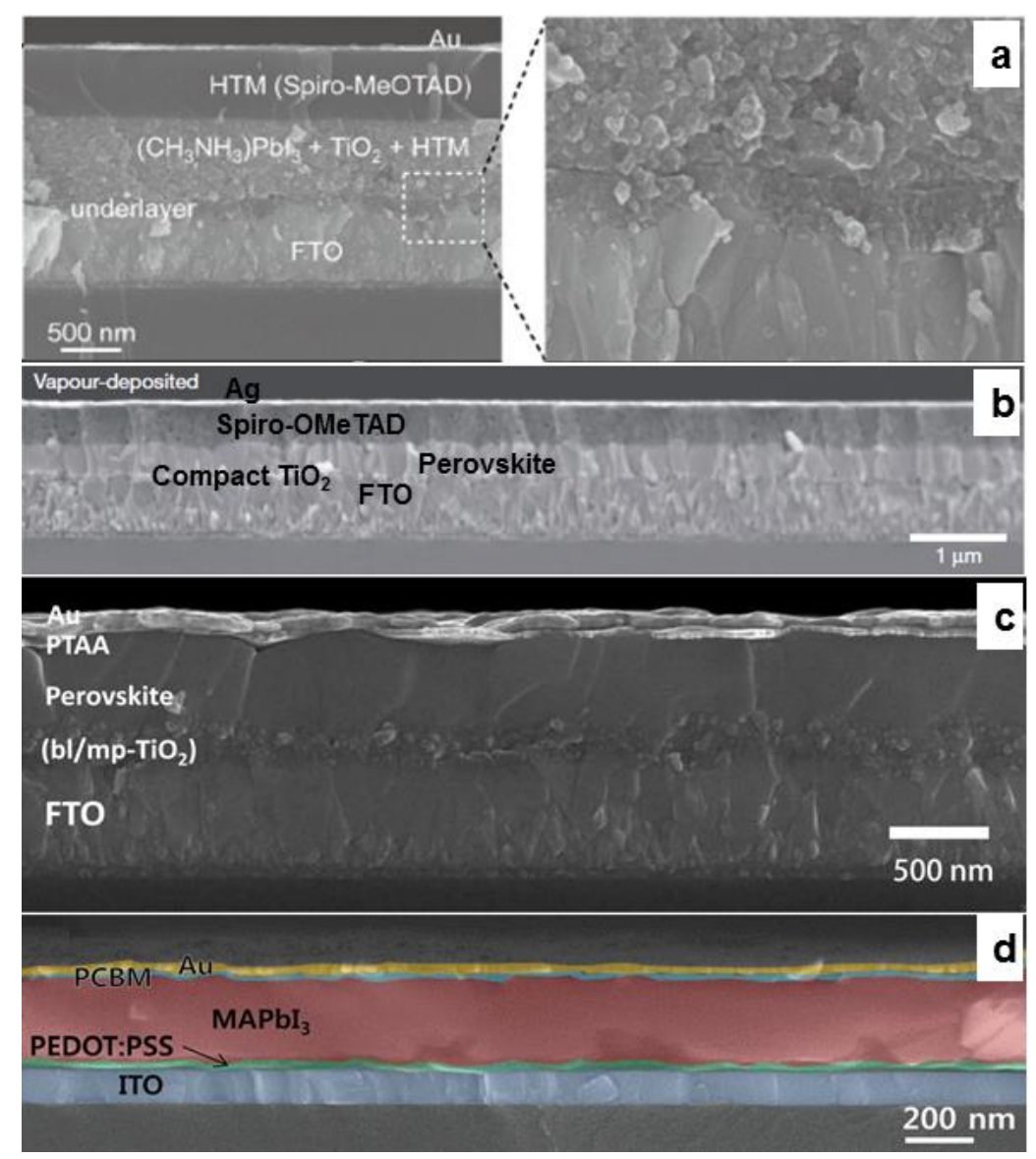

Figure 2. Structural evolution of high-performance perovskite solar cells. Cross-section SEM images of perovskite solar cells in $\mathbf{a}$, mesoscopic ${ }^{9}, \mathbf{b}$, planar $n$-i-p heterojunction ${ }^{12}, \mathbf{c}$, planar/mesoscopic hybrid ${ }^{30}$ and $\mathbf{d}$, inverted $\mathrm{p}$-i-n planar heterojuction structure ${ }^{31}$ (false color) . Figures reproduced with permission from: $\mathbf{a}$, ref 9 , and $\mathbf{b}$, ref 12 , Nature Publishing Group; c, ref 30, AAAS; d, ref 31, RSC. 


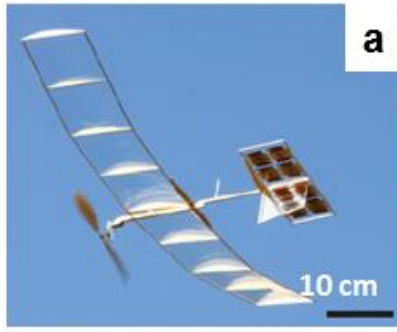

d

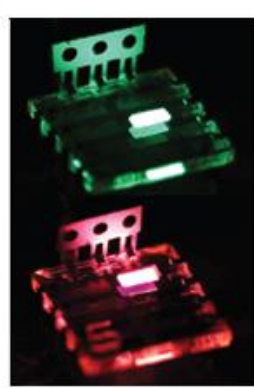

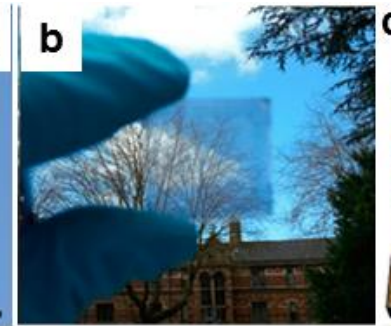

e

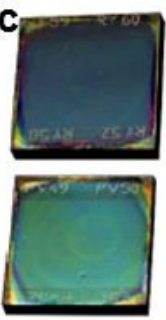

Energy (eV)
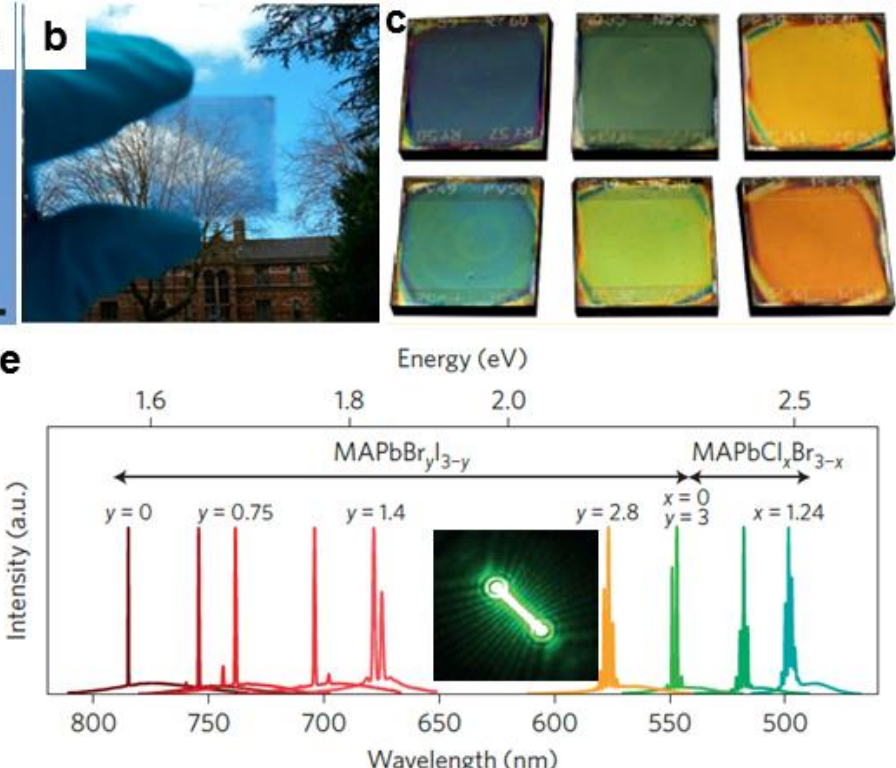

f

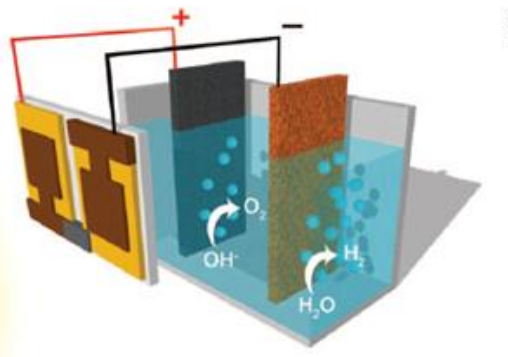

g

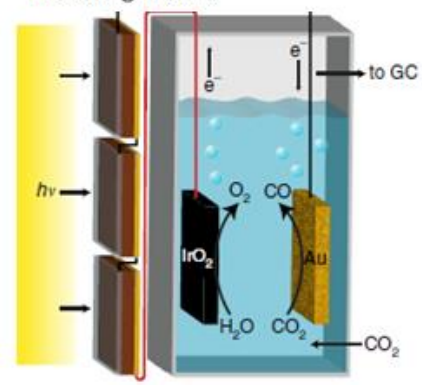

Figure 3. Versatile applications of metal halide perovskites. a, photograph of an model plane powered by perovskite $\mathrm{PVs}^{35}$. $\mathbf{b}$, photograph of a neutral-colored semitransparent perovskite film formed on glass via controlled dewetting ${ }^{36}$. c, photographs of colourful perovskite solar cells incorporating porous photonic crystal scaffolds ${ }^{37}$. d, green and red perovskite light-emitting diodes from ITO/PEDOT:PSS/ $\mathrm{CH}_{3} \mathrm{NH}_{3} \mathrm{PbBr}_{3} / \mathrm{F} 8 / \mathrm{Ca} / \mathrm{Ag}$ and ITO/PEDOT:PSS/ $\mathrm{CH}_{3} \mathrm{NH}_{3} \mathrm{PbBr}_{2} \mathrm{l} / \mathrm{F} 8 / \mathrm{Ca} / \mathrm{Ag}$, respectively ${ }^{40}$. e, widely tunable lasing emission wavelength at room temperature from single-crystal nanowire lasers (inset photograph) of mixed lead halide perovskites ${ }^{43}$. $\mathbf{f}$, schematic diagram of the water-splitting device combining the perovskite PVs $\left(\mathrm{FTO} / \mathrm{TiO}_{2} / \mathrm{CH}_{3} \mathrm{NH}_{3} \mathrm{Pbl}_{3} /\right.$ Spiro-OMeTAD/Au) with NiFe layered double hydroxides/Ni foam electrodes ${ }^{44}$. $\mathbf{g}$, schematic diagram of the sunlight-driven $\mathrm{CO}_{2}$ reduction device combining perovskite PVs $\left(\mathrm{FTO} / \mathrm{TiO} \mathrm{O}_{2} / \mathrm{CH}_{3} \mathrm{NH}_{3} \mathrm{Pbl} / 3\right.$ Spiro-OMeTAD/Au) with an electrochemical cell using oxidized $\mathrm{Au}$ and $\mathrm{IrO}_{2}$ as electrodes ${ }^{46}$. Figures reproduced with permission from: a, ref 35, Nature Publishing Group; b, ref 36, ACS; c, ref 37, ACS; d, ref 40, and e, ref 43, Nature Publishing Group; f, ref 44, AAAS; g, ref 46, Nature Publishing Group. 

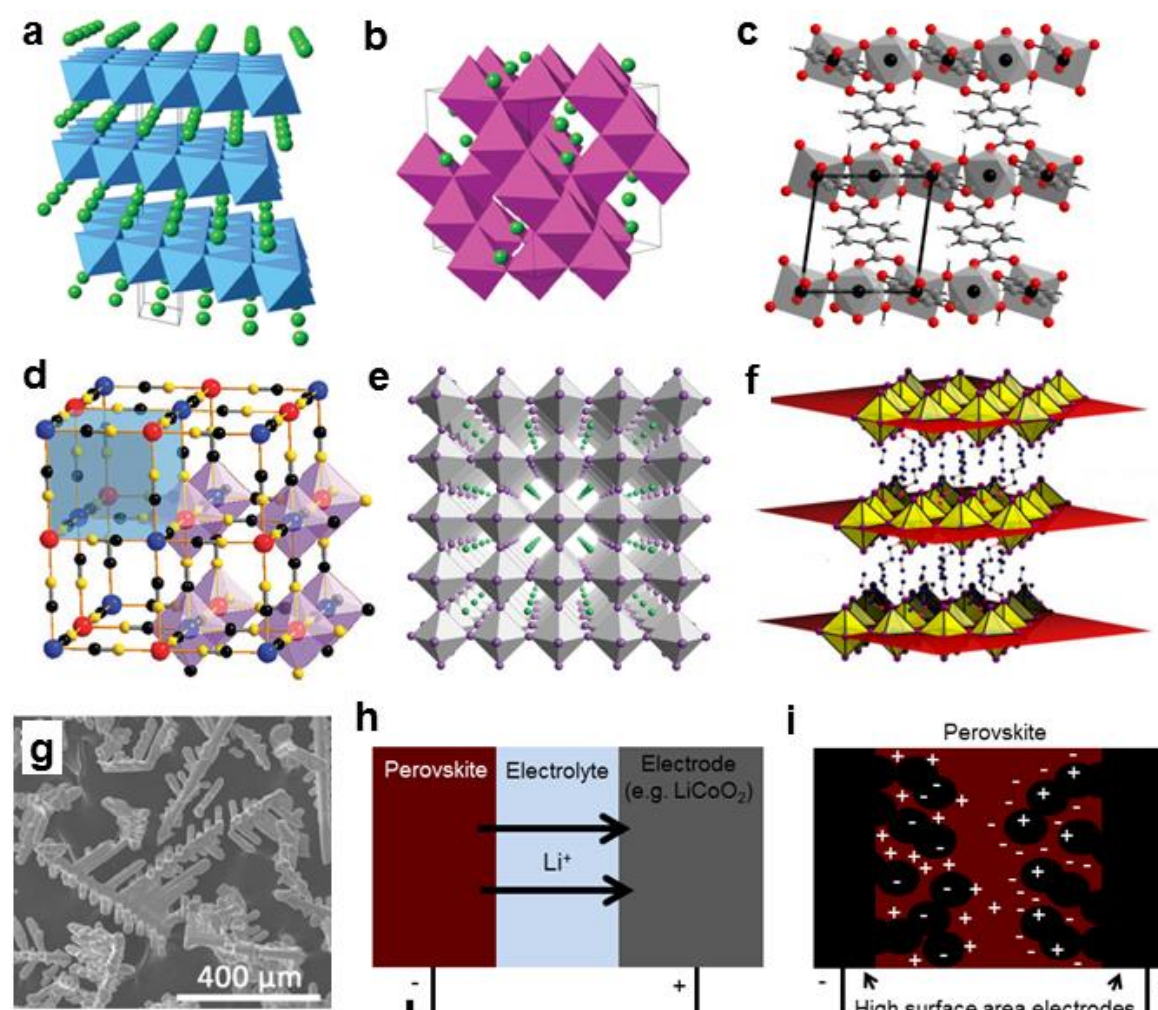

h
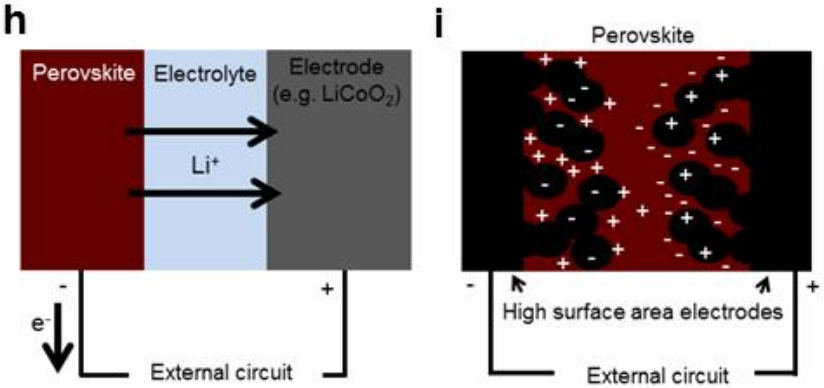

Figure 4. Future directions and opportunities. Crystal structure of ion battery electrode materials: a) layered $\mathrm{LiCoO}_{2}{ }^{65}$; b) cubic spinel $\mathrm{LiMn}_{2} \mathrm{O}_{4}{ }^{65}$; c) Metal-organic framework MIL53(Fe) ${ }^{66}$; d) Prussian blue analogue ${ }^{67}$. Perovskite structures: e) 3D MAPbl $_{3}{ }^{70}$; f) 2D layered butylammonium lead iodide ${ }^{54} . \mathrm{g}$ ) hydrothermally synthesised $\mathrm{MAPbBr}_{3}$ used in the first report of a perovskite battery anode ${ }^{68}$. h) and i) possible architectures for a perovskitebased battery and supercapacitor respectively. The battery is shown in discharging state and the capacitor in charged state. Figures reproduced with permission from: $\mathbf{a} \& \mathbf{b}$, ref $65, \mathrm{RSC}$; c, ref 66, Elsevier B.V.; d, ref 67, RSC; e, ref 70, Nature Publishing Group, f, ref 54, ACS, g, ref $68, \mathrm{RSC}$. 